40-1| 2015

Enjeux contemporains en études irlandaises - In Memoriam Paul Brennan

\title{
L'Église et la modernisation de l'enseignement du second degré en Irlande au début des années soixante
}

Imelda Elliott

\section{(2) OpenEdition}

\section{Journals}

Édition électronique

URL : http://journals.openedition.org/etudesirlandaises/4485

DOI : 10.4000/etudesirlandaises.4485

ISSN : 2259-8863

Éditeur

Presses universitaires de Rennes

Édition imprimée

Date de publication : 30 juin 2015

Pagination : 135-148

ISBN : 978-2-7535-4082-8

ISSN : 0183-973X

\section{Référence électronique}

Imelda Elliott, «L'Église et la modernisation de l'enseignement du second degré en Irlande au début des années soixante ", Études irlandaises [En ligne], 40-1 | 2015, mis en ligne le 30 juin 2017, consulté le 19 avril 2019. URL : http://journals.openedition.org/etudesirlandaises/4485 ; DOI : 10.4000/ etudesirlandaises. 4485 


\title{
L'Église et la modernisation de l'enseignement du second degré en Irlande au début des années soixante ${ }^{1}$
}

\author{
Imelda ELLIOTT \\ Université du Littoral Côte d'Opale
}

\section{Résumé}

Cet article explore des documents des archives de l'Archevêque de Dublin pour analyser le rôle de l'Église et de l'État dans la modernisation de l'enseignement du second degré au début des années soixante. L'auteur présente les propositions faites par l'État pour moderniser l'enseignement du second degré et les documents de l'Église - échanges de courriers et comptes rendus des réunions des évêques - portant sur la réforme de l'enseignement du second degré.

Mots clés : éducation, Église catholique d'Irlande, société et religion

\section{Abstract}

This article explores documents from the Dublin Diocesan Archives to analyse the roles of Church and State in the modernisation of Irish second-level education at the beginning of the sixties. The author presents the State proposals for modernising second-level education and church documents - exchanges of letters and minutes of the meetings of the bishops - dealing with the reform of second-level education.

Keywords: education, Irish Catholic Church, society and religion

Au début des années quatre-vingt-dix, le rôle de l'Église catholique dans l'éducation en Irlande était un sujet tabou que certains des membres éminents du clergé ne souhaitaient pas vraiment aborder. Cependant, je pus découvrir un rapport de commission, inconnu jusque-là, qui aborde le sujet ${ }^{2}$. Le ministre de l'Éducation, P. Hillery ${ }^{3}$, venait de terminer son mandat en tant que président de l'Irlande. Il accepta de répondre à mes questions au cours de plusieurs entre-

1. À travers cet article, je voudrais rendre hommage à Paul Brennan qui joua un très grand rôle dans le développement des études irlandaises en France. Il me fit comprendre l'intérêt d'entreprendre des recherches sur l'Église et l'État dans le système éducatif irlandais alors que mon projet initial portait sur des aspects sociologiques des lycées irlandais.

2. Imelda Bonel-Elliott, "The role of the Duggan Report (1962) in the reform of the Irish education system ", Administration, 43 (3), autumn 1996, p. 42-60.

3. Patrick Hillery fut ministre de l'Éducation de 1959 à 1965. Ensuite, il occupa différents postes ministériels de 1965 à 1972. Il fut Président de la République d'Irlande de 1976 à 1990. 
tiens. J'eus l'opportunité d'entrer en contact avec de nombreuses personnalités du monde éducatif, des ministres, des hauts fonctionnaires, des responsables de l'Église, des dirigeants de syndicats enseignants, des journalistes, prêts à apporter leur concours à mes recherches.

À l'époque, les historiens de l'éducation considéraient que la modernisation de l'enseignement du second degré commençait en 1966 avec l'avènement de la gratuité pour tous. Je voulais démontrer que la nouvelle politique de 1967 n'aurait pas été possible sans les changements mis en place à partir de $1963^{4}$. Aucun chercheur en Irlande n'avait travaillé sur cette époque. J'ai pu ainsi défricher le terrain et essayer d'attirer l'attention sur une période importante dans l'histoire du système éducatif irlandais.

À présent, à la lumière des archives de l'archevêque de Dublin John Charles McQuaid, je voudrais revisiter le début des années soixante afin d'analyser le rôle de l'Église et de l'État dans la modernisation de l'enseignement du second degré pour voir dans quelle mesure le projet de l'État fut limité par le poids de l'Église catholique. Était-ce un projet véritablement révolutionnaire pour l'époque? Si la période, les circonstances l'exigeaient, pourquoi rencontra-t-il des résistances? Si l'Église y était hostile, comment la réforme put-elle s'imposer? Nous allons donc, dans un premier temps, exposer les aspects saillants du système éducatif au début des années soixante. Ensuite, nous étudierons les propositions faites par l'État pour le moderniser. Enfin, nous examinerons les documents de l'Église dans le contexte de la réforme de l'enseignement du second degré.

\section{L'enseignement du second degré au début des années soixante}

En 1963, il existait en Irlande deux types d'écoles du second degré : d'une part l'école secondaire (secondary school), ou lycée classique, une école privée, payante et généralement dirigée par les congrégations religieuses ou les évêques ${ }^{5}$, et d'autre part l'école technique (vocational school), publique, gérée par un comité éducatif professionnel issu des collectivités locales $(V E C)^{6}$. Dans les petites villes, on trouvait souvent trois établissements du second degré distincts : une école secondaire de garçons appartenant au diocèse (diocesan college) ou aux congrégations religieuses, une école secondaire de jeunes filles appartenant aux religieuses (Convent School) et une école technique mixte. Dans les écoles secondaires, afin de pouvoir accéder aux études longues, les élèves, vers quinze ou seize ans, passaient un

\footnotetext{
4. I. Bonel-Elliott, "Lessons from the Sixties: Reviewing Dr. Hillery's Educational Reform ", Irish Educational Studies, vol. 13, Spring 1994, p. 32-45.

5. I. Bonel-Elliott, "1963 Année décisive dans l'enseignement irlandais? ", Éducations anglo-saxonnes : de l'an mil à nos jours I, Amiens, Sterne, 1992, p. 175-190.

6. VECs: Vocational Education Committees.
} 
examen, l'Intermediate Certificate, après trois années d'études secondaires. Celui-ci ouvrait sur le Senior cycle (deux ans de plus) à la fin duquel on passait, à 17 ans, le Leaving Certificate, l'équivalent du baccalauréat français.

Dans les écoles techniques, les élèves suivaient deux années d'enseignement général et d'enseignement technique menant à un diplôme dénommé le Group Certificate donnant accès à l'apprentissage ou à la vie active. Les écoles techniques n'étaient pas autonomes mais faisaient partie d'un groupement d'établissements, correspondant généralement à un comté ou à une grande ville, administré par un comité éducatif comprenant quatorze membres choisis par les collectivités locales placé sous l'égide d'un secrétaire général (Chief Executive Officer ou CEO). Six au moins ne devaient pas être des élus locaux. Les membres devaient représenter les intérêts divers de chaque région : l'éducation, la culture, l'industrie et le commerce. Le secrétaire général exerçait les fonctions de directeur, de secrétaire et de comptable des services éducatifs? ${ }^{7}$. Les écoles techniques n'accueillaient qu'une minorité d'élèves, généralement les plus défavorisés. L'Église catholique avait accepté en 1930 l'existence de ces écoles publiques à la condition que l'on n'y dispense pas un enseignement général long $^{8}$, car elle voulait surtout avoir la mainmise sur l'éducation des classes dominantes?.

Le clergé et les congrégations religieuses catholiques étaient propriétaires de la plupart des écoles secondaires qu'ils contrôlaient. 89 \% des élèves du second degré fréquentaient ces établissements ${ }^{10}$. Comme l'Église catholique prenait l'initiative en matière d'enseignement, l'État n'était pas obligé d'investir dans la construction de bâtiments. Certains chercheurs estiment qu'il y avait un gaspillage énorme. En effet, les écoles étaient petites compte tenu que l'on séparait les enfants par religion, par sexe et même par classe sociale ${ }^{11}$. Certaines régions rurales n'étaient aussi pas desservies du tout ${ }^{12}$.

L'Église catholique irlandaise était farouchement opposée aux écoles mixtes. En 1963, seulement 85 écoles secondaires sur un ensemble de 608 étaient mixtes (soit $14 \%)$. Précisons que la plupart de ces écoles mixtes étaient protestantes et que moins de $10 \%$ des enfants catholiques fréquentaient ce type d'établissement ${ }^{13}$. Ces chiffres dissimulent toutefois des différences régionales : à Dublin, seulement 6,8 \% des écoles secondaires étaient mixtes, dans les comtés de l'Ulster $31 \%$ et

7. OECD Survey Team, Investment in Education, Stationery Office, Dublin, 1966.

8. S. O. Buachalla, Education Policy in Twentieth Century Ireland, Dublin, Wolfhound Press, 1988.

9. B. Titley, Church, State and the Control of Schooling in Ireland, Dublin, Gill and Macmillan, 1983.

10. J. Whyte, Church and State in Modern Ireland. 1923-70, Gill and Macmillan, Dublin, 1971.

11. B. Titley, op. cit.

12. I. Elliott, "L'évolution du rôle de l'Église dans l'enseignement en Irlande ", dans J. Lalouette, X. Boniface, J.-F. Chanet et I. Elliott, (eds.), Les religions à l'École : Europe et Amérique du Nord XIXe-XXI siècles, Paris, Letouzey \& Ané, 2011, pp. 29-40.

13. D. Akenson, A Mirror to Kathleen's Face: Education in Independent Ireland, 1922-60, Montreal, McGill-Queen's University Press, 1975. 
dans le Connacht $25 \%{ }^{14}$. La séparation des garçons et des filles était la norme pour la majorité des élèves surtout en milieu urbain. En effet, l'archevêque de Dublin, J.C. McQuaid, interdisait la mixité dans son diocèse. Il refusait même d'assister à l'inauguration d'une école technique si elle était mixte ${ }^{15}$. En revanche, d'autres évêques, dans les régions rurales, acceptaient cette situation dès lors que cela s'avérait indispensable pour avoir des effectifs suffisants ${ }^{16}$. Ainsi, l'Église pouvait mener sa politique de non-mixité avec plus d'efficacité dans les centres urbains, notamment à Dublin, et devait accepter des compromis dans les régions moins urbanisées.

Au début des années soixante, la Commission Investment in Education, sous l'égide de l'OCDE, œuvrait sous la direction de Patrick Lynch, alors professeur d'économie à University College Dublin. Le ministre reçut les premières conclusions de ce groupe de travail dès le début de 1963. Une seconde commission de fonctionnaires (surtout des inspecteurs), créée en 1962, étudia le système éducatif et les réformes possibles. La composition en était confidentielle, inconnue des administrateurs des écoles, des enseignants et du public. Il est vraisemblable que le ministre considéra que, pour entreprendre une réforme, il valait mieux travailler dans le secret, compte tenu de l'échec du travail produit par le Conseil de l'Éducation qui, en ce qui le concerne, soutint le statu quo. La commission, sous la présidence de Maurice Duggan, rédigea un rapport ${ }^{17}$. Elle devait surtout considérer les aspects sociaux d'une réforme, le rapport de l'OCDE portant, lui, sur les aspects économiques.

Le fait que le rapport de la commission Duggan fut rédigé par des fonctionnaires est intéressant à plus d'un titre dans la mesure où ces derniers étaient souvent accusés d'être opposés aux changements ${ }^{18}$. Cependant, le ministre ne pouvait pas compter sur la plupart des hauts fonctionnaires, surtout sur les inspecteurs généraux, hostiles aux innovations. Il fit donc appel à des inspecteurs plus jeunes et dut faire beaucoup d'efforts pour encourager ces fonctionnaires du ministère de l'Éducation, démoralisés de se voir constamment opposer à leurs tentatives de changements des fins de non-recevoir, si bien qu'un inspecteur surnomma le ministère de l'Éducation (oideachas) le ministère du désespoir (éadochas), jouant sur la ressemblance entre les deux mots en gaélique ${ }^{19}$.

Au mois d'octobre 1962, lors de son discours à la première réunion du groupe d'étude Investment in Education, le ministre souligna également, d'un point de

\footnotetext{
14. Investment in Education, op. cit.

15. Entretien avec Soeur E. Randles.

16. D. Akenson, op. cit.

17. Department of Education, Report on Post-Primary Education, 1962.

18. I. Bonel-Elliott, "The role of the Duggan Report (1962) in the reform of the Irish education system ", op. cit. 19. Entretien avec P. Hillery.
} 
vue économique, le problème du manque de personnel qualifié pour faire face à l'expansion industrielle alors en cours. Comme on avait été amené à l'époque à prendre véritablement conscience que l'investissement dans l'Éducation pouvait être au moins aussi productif que dans le domaine industriel, cela permit au ministre de solliciter des fonds pour le système éducatif ${ }^{20}$. De la même manière, P. Hillery insista sur la nécessité pour l'Irlande de le moderniser en vue de l'entrée du pays dans le marché commun.

\section{Le projet de modernisation de l'enseignement du second degré en 1963}

Lors de sa conférence de presse en mai 1963, P. Hillery exposa les objectifs de sa réforme. Sa volonté était que tous les enfants puissent bénéficier d'un enseignement du second degré adapté à leurs aptitudes et à leurs capacités ${ }^{21}$. L'élève d'une comprehensive school devait préparer l'Intermediate Certificate (l'équivalent en France du Brevet des Collèges). Dans le cas de son obtention, il pourrait alors, selon ses aptitudes, poursuivre des études secondaires classiques ou techniques. Le projet de programme des comprehensive schools comportait un tronc commun avec les matières telles que le gaélique, l'anglais, les mathématiques et des matières techniques (le dessin ou l'enseignement manuel) auxquelles s'ajoutaient les options comme l'histoire, la géographie, le latin, une ou plusieurs langues étrangères, les sciences, l'agronomie, les beaux-arts, le chant, etc. Le ministre considérait que les cours d'instruction religieuse allaient de $\operatorname{soi}^{22}$.

Le ministre décida également d'allonger la scolarité dans les écoles techniques en la faisant évoluer de deux à trois ans. Possibilité était donnée aux élèves de passer le même examen, l'Intermediate Certificate, que leurs camarades des écoles secondaires. À cette fin, le ministère élargit les programmes pour cet examen en $\mathrm{y}$ incluant des matières techniques. Les programmes et les examens communs à toutes les écoles du second degré assureraient une scolarité équivalente dans tous les établissements.

Afin de mettre en ouvre la politique d'un système polyvalent, le ministre décida de créer des écoles polyvalentes dans les secteurs où n'existaient pas d'établissements d'enseignement du second degré, de mettre en place un système de transport scolaire, d'uniformiser les programmes et les examens. Ces écoles polyvalentes, initialement, devaient être des collèges uniques. Le discours du ministre

20. Speech delivered by the Minister for Education at the first meeting of the Steering Committee of Investment in Education, 31 octobre 1962.

21. P. Hillery, « Press Conference » Dublin : Department of Education, 20 mai 1963.

22. Ibid. 
en 1963 étant volontairement assez vague, le projet initial subit plusieurs adaptations avant sa mise en œuvre définitive.

Le ministre annonça sa nouvelle politique dans une conférence de presse à l'intention de la population et des députés avant même le débat parlementaire et la présentation des prévisions budgétaires annuelles du ministère de l'Éducation. Agir de la sorte, avant même la mise au point des détails, était pour P. Hillery une manière d'entraîner dans son sillage, en les contraignant, le gouvernement et les fonctionnaires. Sa réforme s'inscrivait dans la perspective de la politique générale du gouvernement. Par conséquent, ses collègues du Cabinet ministériel la considérèrent favorablement. Parmi les objectifs du deuxième programme pour l'expansion économique, apparaissait l'enseignement en tant qu'investissement ${ }^{23}$. Le ministre n'élabora pas de projet de loi d'orientation sur l'Éducation, et ce en dépit de la pression de l'opposition sur le sujet et des questions parlementaires ${ }^{24}$. Il évoqua simplement la probabilité d'un projet de loi sur les écoles techniques, indispensable pour permettre aux comités éducatifs professionnels de participer à l'administration des nouvelles écoles polyvalentes. Même si ce discours laissait encore beaucoup de points d'interrogation sur de nombreux détails, P. Hillery annonça clairement la création d'un nouveau type d'établissement.

Afin de développer le contenu de la réforme, P. Hillery travaillait surtout avec deux hauts fonctionnaires qui jouèrent un rôle important dans l'élaboration du projet :

From that on it was Terry Raifeartaigh, Sean MacGearailt and myself. We met regularly every day. They were running the Department which was constantly under fire for all sorts of things. We were talking about it every day but anything well written was written by Terry O'Raifeartaigh. T. O'Raifeartaigh was very well educated, very well learned, very wise. You'd know you were right if he was staying with you. He wouldn't let you go wrong. He knew education inside out. MacGearailt made things go. We were always together and anything I knew about education I learned it from $\mathrm{him}^{25}$.

Avant de mettre en place des commissions, le ministre avait pu cerner un certain nombre de défauts du système éducatif. Selon lui, l'un des plus préoccupants était d'ordre social dans la mesure où les enfants défavorisés ne pouvaient pas bénéficier d'un enseignement secondaire ${ }^{26}$. P. Hillery fut influencé par son

23. Second Programme for Economic Expansion, Dublin: Stationery Office, 1964.

24. Dáil Debates, vol. 203, 28 mai 1963.

25. Entretien avec P. Hillery.

26. "Address of P. Hillery to the Dublin Comhchomhairle of Fianna Fail in Clery's Restaurant », 28 March 1961. 
expérience personnelle, conscient qu'il avait toujours été qu'on n'offrait aucune chance aux jeunes de milieux défavorisés ${ }^{27}$.

Les propositions du ministre peuvent se résumer de la façon suivante : créer des écoles polyvalentes, mettre en place un système de transport dans les secteurs où il n'existait pas d'enseignement secondaire et donner la possibilité aux élèves des collèges techniques de passer les mêmes examens que les élèves des écoles secondaires. Les programmes des nouvelles écoles comprenaient l'enseignement général, l'enseignement technique et la religion. L'État prendrait en charge les coûts de construction et de fonctionnement ainsi que les salaires des enseignants. Le ministre aspirait à une démocratisation de l'enseignement à la fois pour des raisons de justice sociale mais aussi pour contribuer au développement économique de la nation. La création des écoles polyvalentes était une manière d'assurer l'essor de l'enseignement du second degré. En outre, cette proposition s'accordait en tout point avec l'esprit même de la politique éducative des années soixante dans les autres pays occidentaux où le collège unique émergeait comme un modèle dominant ${ }^{28}$. Toutefois, en Irlande, il était nécessaire de négocier avec le groupe le plus puissant dans l'enseignement secondaire : l'Église catholique.

\section{L'Église et la réforme de l'enseignement du second degré}

Dès 1962, lors d'une réunion en assemblée générale, les évêques évoquèrent le projet de création des écoles polyvalentes. Ils craignaient la mise en place d'écoles secondaires d'État et s'inquiétaient pour l'avenir des petits séminaires (diocesan colleges) privés ainsi que pour celui des écoles privées appartenant aux congrégations. Ils décidèrent d'envoyer les deux évêques en charge de l'éducation ${ }^{29}$ s'entretenir avec les hauts fonctionnaires du ministère de l'éducation ${ }^{30}$. Estimant qu'il s'agissait d'écoles mixtes non-confessionnelles, J.C. McQuaid, Archevêque de Dublin, considérait pour sa part que la création d'écoles d'État était contraire à la constitution.

The proposed scheme seems to me open to the gravest objections. It is, in fact, in my view, contrary to the provisions of the Constitution, Article 42, especially no. 3 and no. 4. The Department, instead of aiding the preference of Catholic parents for fully Catholic schools, by helping the parents and Church

27. Entretien avec P. Hillery.

28. N. Barber, Comprehensive Schooling in Ireland, Dublin, ESRI, 1989.

29. V. Hanly, évêque d'Elphin et J. Fergus, évêque d'Achonry.

30. Minutes of the general meeting of the Irish hierarchy, 1er octobre 1962, Reference XV/22/1-20, McQuaid Papers, Dublin Diocesan Archives (DDA). 
to establish such schools, is proposing to establish, with taxpayers money, State secondary schools, non-denominational, co-educational ${ }^{31}$.

Vincent Hanly, évêque d'Elphin, fit remarquer que la mise en place d'un système d'enseignement polyvalent était inévitable. Il souhaitait que la hiérarchie participe à la rédaction de la nouvelle loi :

In my humble opinion the bishops should enter the field immediately as other parties are getting active. If possible the bishops should take a hand in framing the proposals for new legislation as the authorities appear to be frien$d l y$ and anxious to invite suggestions. Some form of comprehensive education - free for all - is due to come and we should be ready to welcome it and direct it into proper channels ${ }^{32}$.

L'Archevêque de Dublin déclara que l'État n'avait pas le droit d'utiliser l'argent des contribuables en vue de créer des écoles publiques non-confessionnelles qui seraient contraires aux vœux des familles catholiques. Pour lui, le financement de l'État ne donnait aucun droit de contrôle à ce dernier sur les programmes, la gestion et les personnels des écoles.

The State has not the function nor the right to establish a type of school to which the Catholic parents shall, in effect, be obliged, directly or indirectly, to send their children, contrary to their conscience, and lawful preference. The State has not the right to use the taxpayers money to establish such schools. Neither has the State the right to control the curriculum or management or staffing of a school because it contributes aid from the taxpayers' money ${ }^{33}$.

J.C. McQuaid souhaitait même bloquer le projet de modernisation du ministre : "Would it not be better to block the proposals before they reach us lest a wrong scheme be advanced ${ }^{34}$ ? " La crainte de V. Hanly, évêque d'Elphin, était, d'une part, que les parents veuillent que leurs enfants aient accès à un enseignement secondaire polyvalent et, d'autre part, que toutes les écoles techniques publiques existantes deviennent des écoles polyvalentes gratuites entrant alors en concurrence avec les écoles privées payantes. Le secrétaire général du ministère de l'éducation le rassura en soulignant que l'enseignement du latin était indispensable pour détenir le statut d'école secondaire ${ }^{35}$.

Au mois de février 1963, T. O’Raifeartaigh, secrétaire général du ministère de l'Éducation, se déplaça pour voir les deux évêques en charge des questions éduca-

31. Letter from Archbishop of Dublin to Bishop of Elphin 5 janvier 1963, XV/23/12, McQuaid Papers, DDA. 32. Letter from Bishop of Elphin to Archbishop McQuaid, 4 janvier 1963, XV/23/11, McQuaid Papers, DDA. 33. Letter from Archbishop of Dublin to Bishop of Elphin, 5 janvier 1963, XV/23/12, McQuaid Papers, DDA. 34. Notes écrites à la main par l'Archevêque de Dublin (18 janvier 1963), XV/23/14, McQuaid Papers, DDA. 35. Bishop of Elphin to Archbishop of Dublin, 18 février 1963 XV/23/16, McQuaid Papers, DDA. 
tives afin de leur expliquer le projet de réforme et la mise en place d'une dizaine d'écoles polyvalentes avec un service de transport scolaire. Le ministère prévoyait un conseil d'administration de trois membres représentant le ministère, les collectivités locales et les évêques ${ }^{36}$. James Fergus, évêque d'Achonry, avait compris que le but principal de la visite du secrétaire général du ministère était de les informer que le ministre allait faire une déclaration concernant le projet de modernisation de l'enseignement du second degré et que ce dernier souhaitait dire que le ministère avait consulté les autorités ecclésiastiques. Les représentants des évêques demandèrent que le ministre n'évoque pas l'Intermediate Certificate pour les écoles techniques. Le secrétaire général expliqua que l'urgence venait de la nécessité de publier le projet avant la publication par le parti travailliste d'un document sur l'éducation ${ }^{37}$.

Larchevêque de Dublin obtint, semble-t-il, assez facilement un rendez-vous avec le secrétaire général du ministère de l'Education ${ }^{38}$. Ce dernier sut rassurer l'archevêque, affirmant que l'Église n'avait rien à craindre pour ses écoles existantes. Dans une lettre faisant le compte rendu de l'entrevue, l'archevêque évoqua la nature confessionnelle des nouveaux établissements et le fait qu'ils seraient gérés par les prêtres, éléments qui apportaient selon lui les garanties nécessaires quant aux enseignants, aux programmes et à la discipline :

I had a long conversation with Dr. O'Raifeartaigh. On the whole, I do not think that we have to fear for our existing schools [...]. The definitely denominational, non co-educational character of the comprehensive school, with management by the Parish clergy, gives us the necessary guarantee in regard to Catholic teachers and curriculum and discipline $e^{39}$.

Le 24 avril 1963, les deux évêques chargés des questions éducatives se rendirent au ministère de l'Education et réussirent à obtenir une entrevue avec le secrétaire-général adjoint (S. MacGearailt) en l'absence du ministre et du secrétaire général. Ils précisèrent le point de vue des évêques et les points d'achoppement. Ils revendiquaient des écoles séparées et des moyens de transport séparés pour les filles et les garçons : "The deputation impressed upon Mr. MacGearailt [...] that the Bishops would oppose anything in the way of mixed education as well as any system of transport where boys and girls would be obliged to travel together ${ }^{40}$." Dans un mémorandum apparemment rédigé par les évêques, on trouve : "There

36. Bishop of Achonry to Archbishop of Dublin 20 février 1963, McQuaid Papers, DDA.

37. Ibid.

38. Archbishop of Dublin to Bishop Fergus, 23 février 1963, McQuaid Papers, DDA.

39. Ibid.

40. Interview with the Department of Education 24 avril 1963, XV/23/19, McQuaid Papers, DDA. 
shall be separate schools for boys and girls. Boys and girls should not be allowed to travel on the same School Bus ${ }^{41} \ldots$ ".

Les évêques souhaitaient que l'Intermediate Certificate pour les écoles techniques ne soit pas l'équivalent de celui des écoles secondaires ${ }^{42}$. Le haut fonctionnaire sut apaiser les évêques. Il précisa que le ministre allait faire une déclaration au parlement et qu'il n'aurait pas le temps de les consulter auparavant. Les évêques demandèrent qu'un exemplaire du discours soit remis à l'archevêque de Dublin. Ils revendiquèrent également des financements pour les bâtiments des écoles secondaires ${ }^{43}$.

Ils insistèrent sur l'insuffisance des subventions accordées en les comparant à celles des lycées en Angleterre, et sur le fait qu'il était du devoir de l'État de faire les efforts financiers nécessaires pour les écoles catholiques. Ils ajoutèrent que les faibles frais de scolarité qui y étaient pratiqués constituaient ni plus ni moins une forme de bourse pour les lycéen ${ }^{44}$.

Le 28 juin 1963, soit un mois après le discours de P. Hillery, les évêques obtinrent un entretien avec ce ministre, le secrétaire général du ministère T. O'Raifeartaigh et le secrétaire adjoint $S$. MacGearail $t^{45}$. Les évêques envoyèrent leur compte rendu au secrétaire général pour avoir l'accord du ministre de l'Éducation sur le contenu. Ils tenaient à être informés sur le nombre de nouvelles écoles polyvalentes prévues. Pour eux, l'intervention de l'État dans l'enseignement classique secondaire et l'autorisation accordée aux écoles techniques de devenir des lycées classiques constituaient un changement radical. Leurs inquiétudes tournaient autour de quatre points : la crainte de voir des écoles mixtes (garçons et filles), l'absence de projet de financement des bâtiments des écoles secondaires privées, la gestion et les programmes des nouveaux établissements ${ }^{46}$.

Dans son communiqué de presse, le ministre exposa la politique générale tout en voulant se ménager une marge de manœuvre afin de pouvoir tenir compte des réactions des groupes de pression. Une étape suivante était prévue au cours de laquelle les hauts fonctionnaires consulteraient l'évêque des diocèses concernés par l'implantation d'une nouvelle école. Les évêques avaient cru comprendre qu'il ne s'agirait pas d'écoles mixtes mais d'écoles de garçons ${ }^{47}$. Ils envoyèrent leur compte rendu à T. O'Raifeartaigh pour avoir la confirmation qu'il s'agissait du reflet exact

\footnotetext{
41. Memorandum on Irish Secondary Education, XV/23/23, McQuaid Papers, DDA. Ce mémorandum daterait de 1963, aucune date n’apparaissant sur le document.

42. Interview with the Department of Education 24 avril 1963, XV/23/19, McQuaid Papers, DDA.

43. Ibid.

44. Memorandum on Irish Secondary Education, XV/23/23, McQuaid Papers, DDA.

45. Memorandum of interview with Minister concerning the New Schools Scheme (28 juin 1963), Standing Committee 21 septembre 1963, McQuaid Papers, Hierarchy Meetings Maynooth, DDA.

46. Ibid.

47. Ibid.
} 
des discussions. Ce dernier étant en congé, c’est le secrétaire adjoint S. MacGearailt qui répondit le 26 juillet 1963, précisant que même si le ministre prévoyait un plus grand besoin d'écoles de garçons dans les zones concernées, il serait erroné de dire qu'il n'envisageait que des écoles de garçons. Pour ce qui concerne la gestion, le ministre prévoyait un conseil de trois personnes représentant l'évêque du diocèse, le ministère et les comités éducatifs professionnels issus des collectivités locales (VEC). Le représentant de l'évêque serait le président de ce conseil d'administration, ce qui constituait un pouvoir important pour l'Église. Quant aux postes d'enseignants, le ministre et les hauts fonctionnaires réussirent à imposer une sorte de concours puisqu'il fallait publier les postes vacants et que la commission de sélection devait recruter la personne la plus qualifiée. Chaque nomination était soumise à l'autorisation du ministère mais le président du conseil d'administration (l'évêque ou son représentant) avait un droit de veto sur la base de la foi et des mœurs, et sur cette base uniquement ${ }^{48}$.

Les évêques qui étaient à Rome pour les réunions du Concile de Vatican II écrivirent au ministre afin de recueillir davantage de précisions sur la réforme ${ }^{49}$. Ils proposèrent que le secrétaire général du Comité Educatif Professionnel représente chaque comité. Dans la gestion des écoles, leur préférence allait à un travail avec les administratifs plutôt qu'avec les politiques. Le ministre indiqua que les postes d'enseignants seraient ouverts aux laïcs et aux religieux. Il espérait ainsi favoriser les promotions pour les laïcs, ce qui n'était pas possible dans les écoles secondaires. Concernant la question des écoles mixtes, le ministre donnait l'impression d'aller dans le sens des évêques, déclarant "Normally there would be separate schools for boys and girls. It may happen, however, that in an area of very scattered population it may be necessary to erect a mixed school ${ }^{50} "$. Le ministère étudia la possibilité d'augmenter les financements des écoles secondaires. Le ministre proposa que la formation religieuse des élèves dans les écoles techniques fasse partie intégrante des programmes comme cela était prévu dans les écoles polyvalentes ${ }^{51}$.

Le 5 décembre 1963, les deux évêques chargés de l'éducation eurent un entretien avec P. Hillery, T. O’Raifeartaigh et S. MacGearailt pour examiner le projet des nouvelles écoles ${ }^{52}$. Les évêques, dans leur compte rendu, indiquèrent que le ministre et les hauts fonctionnaires exigeaient que le ministère soit un trustee ${ }^{33}$

48. The New Post-Primary Schools Scheme, Second Memorandum from the Bishops of Elphin and Achonry (on meeting of $28^{\text {th }}$ June 1963), XV/23/66, McQuaid Papers, DDA.

49. Letter from the Bishop of Achonry in the Irish College in Rome 29 octobre 1963, XV/23/77, McQuaid Papers, DDA.

50. Letter from Minister of Education to Bishop of Achonry 20 novembre 1963, XV/23/78, McQuaid Papers, DDA.

51. Ibid.

52. Memorandum - The Hillery Scheme, 5 décembre 1963, XV/23/73, McQuaid Papers, DDA.

53. Trustee est un terme venant du droit anglais, signifiant que ces personnes sont chargées de gérer ces biens dans l'intérêt de la personne morale qui les nomme. Voir R. David, Le droit anglais, Paris, PUF, 1991. 
(propriétaire-administrateur) afin que ce dernier ait l'assurance que les bâtiments ne puissent jamais être employés à d'autres fins que des fins éducatives. Le ministre reconnaissait la nécessité d'une augmentation des financements des écoles secondaires et la présence de l'enseignement de la religion dans les écoles polyvalentes et techniques ${ }^{54}$.

Lors de la commission permanente des évêques du 7 janvier 1964, ces derniers estimèrent, pour ce qui concerne la gestion des écoles polyvalentes, qu'ils pouvaient coopérer avec le ministère compte tenu des assurances obtenues auprès du ministre de l'éducation ${ }^{55}$. Le 7 avril 1964, au cours de la commission permanente, les évêques soulignèrent un changement de politique du ministère : ce dernier ciblait un minimum de 300 élèves par école polyvalente en lieu et place des 150 évoqués dans le discours de mai 1963. Les évêques craignaient la généralisation d'écoles mixtes répondant à un objectif d'établissements à effectifs plus élevés. En juin 1964, lors de l'assemblée générale des évêques, ces derniers prirent la décision de coopérer avec le ministère dans la mise en œuvre des écoles polyvalentes. Chaque évêque avait la latitude de négocier pour les écoles de son diocèse. L'épiscopat était toujours hostile aux écoles mixtes, sauf lorsque c'était incontournable ${ }^{56}$.

Si l'on se réfere aux documents d'archives, les discussions entre les évêques et le ministre de l'Education s'étalèrent sur une période d'environ deux années, de 1962 à 1964. Si le rôle du ministre fut primordial, il ne faudrait toutefois pas sous-estimer celui, tout aussi important, des hauts fonctionnaires. En effet, ces derniers obtinrent que l'argent public dépensé pour les écoles polyvalentes le soit à l'usage exclusif et permanent d'établissements devant conserver leur caractère éducatif ${ }^{57}$. Les enseignants des écoles polyvalentes seront dès lors recrutés par concours après publication des postes et aucun poste ne sera réservé pour les religieux ${ }^{58}$. Si les évêques crurent un moment qu'il s'agirait d'écoles séparées pour les filles d'un côté et les garçons de l'autre, il est fort probable que les hauts fonctionnaires ne les aient pas contredits et soient restés volontairement imprécis. Le ministère créa des écoles mixtes. Les évêques les acceptèrent dans la mesure où elles se trouvaient dans des zones rurales non desservies et aussi parce qu'ils obtinrent de l'État des garanties de financement pour les bâtiments des écoles secondaires privées. Par ailleurs, la présidence du conseil d'administration de l'école polyvalente accordée au représentant de l'évêque dut faciliter l'acceptation

54. Memorandum - The Hillery Scheme, 5 décembre 1963, XV/23/73, McQuaid Papers, DDA.

55. Standing Committee, 7 janvier 1964, XV/24/32, McQuaid Papers, DDA.

56. Minutes, General Meeting of the Irish Hierarchy 24/25 June 1964, WX/24/1-28, McQuaid Papers, DDA.

57. Depuis les années 1990, les bâtiments de certaines écoles secondaires privées financés en grande partie par l'État sont vendus sans que ce dernier puisse être indemnisé au nom des contribuables.

58. Lors de la mise en place des écoles communautaires dans les années 1970, les congrégations obtinrent des postes d'enseignants réservés. Voir : I. Bonel-Elliott, "L'école communautaire en Irlande ", Educations anglosaxonnes: de l'an mil à nos jours II, Amiens, Sterne, 1995, p. 139-163. 
des nouveaux établissements par l'Église. Les écoles étaient confessionnelles mais c'était la norme en Irlande à cette époque.

Sœur Jordana, présidente de l'association nationale représentant les congrégations féminines (CCSS) de 1961 à 1970, dans un entretien qu'elle m'accorda, souligna le rôle important des hauts fonctionnaires. Selon elle, ils étaient suffisamment fins pour être à même de jouer tout un jeu, y compris même celui du « rôle de l'imbécile». Mais au bout du compte, les représentants de l'Église et de l'État surent se respecter et purent trouver un terrain d'entente :

Terry O'Raifeartaigh could play the idiot. But there was a difference between playing the idiot and being the idiot and if you don't know your people, you can be led astray. But Sean MacGearailt. Some people called him a hatchetman. I was secretary to the Conference of Convent Secondary Schools until 1961/62 and then I became president. I was president for nine years and during that period all these things happened. I was involved with many ministers [...]. I remember all of these with great affection and the secretaries to the Department are terribly important. I used to say, the ministers come and go and the Department was on forever and the secretaries are very important [...]. We spoke the same language and understood one another and we could agree and disagree and yet be friends 59 .

Au début des années soixante, l'Église catholique avait un quasi monopole sur l'enseignement secondaire. Le ministre de l'Education ne pouvait pas moderniser le système éducatif sans consulter l'épiscopat. Comme l'Église semblait être hostile aux réformes, il était commode pour le ministère des Finances de se servir de cette opposition afin d'éviter toute augmentation des financements accordés à l'éducation ${ }^{60}$. Compte tenu que le projet de modernisation avait le soutien du Premier Ministre, des médias et des parents, les évêques n'eurent pas d'autre issue que de chercher à négocier et ainsi obtenir ce qui leur semblait essentiel. La réforme fut mise en place. P. Hillery introduisit le principe d'écoles publiques d'État et celui de l'accès pour tous à l'enseignement du second degré, avec les mêmes programmes et examens dans tous les établissements. L'Église catholique sut âprement protéger ses intérêts, notamment la nature confessionnelle des écoles polyvalentes, la présidence des conseils d'administration de ces dernières ainsi que l'instruction religieuse dans tous les établissements du second degré.

L'État parvint à imposer des écoles mixtes alors que les évêques demandaient des écoles séparées. De plus, le transport scolaire réunissait les deux sexes dans le même véhicule alors que l'épiscopat exigeait des bus distincts pour les filles et pour les garçons. En 1962, les évêques étaient opposés aux réformes proposées par

59. Entretien avec Sour Jordana.

60. Entretien avec P. Hillery. 
le ministre mais quand les changements s'avérèrent incontournables, ils s'adaptèrent et réussirent à obtenir que les nouveaux établissements soient catholiques et que l'État finance les bâtiments des écoles secondaires privées. Les projets de réforme du système éducatif au début des années soixante furent limités par le poids de l'Église catholique qui exerça une influence sur la politique de l'enseignement du second degré, encore et toujours perceptible dans les années qui suivirent. Des éclaircissements et des informations supplémentaires sur le rôle des évêques dans la modernisation du système éducatif irlandais au début des années soixante apparaissent à la lumière de l'analyse des rapports de comptes rendus de réunions des évêques et à celle des échanges de courrier de l'épiscopat. 